\title{
INTERDISCIPLINARY APPROACH IN TREATMENT OF ENDODONTIC- PERIODONTAL LESION : ACASE REPORT
}

\author{
Somanath G. ${ }^{2}$, George G. ${ }^{2}$, Sinha J.N. ${ }^{3}$, Gautam V.
}

\begin{abstract}
Endodontic periodontal combined lesion is a clinical dilemma because making a clinical diagnosis and deciding a prognosis are difficult. The treatment of endodontic periodontal lesion combined requires both endodontic therapy and periodontal regenerative procedures.

This case report evaluates the prognosis of endo- perio lesion which was first treated with conventional endodontic therapy and then followed by periodontal therapy. At a three month post operative evaluation there was gain of clinical attachment level and reduction in probing depth with radiographic evidence of substantial alveolar bone gain.
\end{abstract}

KEYWORDS: Endodontic peiodontal lesion, Open debridement, Root canal treatment

1. Post Graduate Resident, Department of Conservative Dentistry and Endodontics, Universal College of Medical Sciences \& Dental Surgery, Bhairahawa, Nepal

2. Post Graduate Resident, Department of Periodontics and Oral Implantology, Universal College of Medical Sciences \& Dental Surgery, Bhairahawa, Nepal

3. Professor \& Head, Department of Periodontics and Oral Implantology, Universal College of Medical Sciences \& Dental Surgery, Bhairahawa, Nepal

4. Associate Professor, Department of Conservative Dentistry and Endodontics, Universal College of Medical Sciences \& Dental Surgery, Bhairahawa, Nepal

For Correspondence

Dr. Geethu Somanath,

Post Graduate Resident,

Department of Conservative Dentistry and Endodontics, Universal College of Medical Sciences \& Dental Surgery, Bhairahawa, Nepal

E-mail: geethusomanath@gmail.com 


\section{Figure 1: Pathways of communication,IJDR,2010,21:4:579-585}

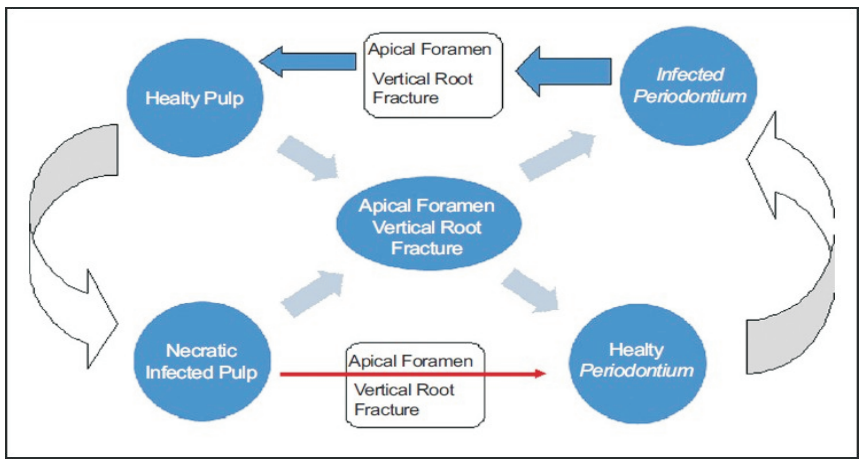

Simon, Glick and Frank (1972) classified endo-perio lesions based on etiology, diagnosis, prognosis and treatment.

- Primary endodontic lesion

- Primary endodontic lesion with secondary periodontal involvement

- Primary periodontal lesion

- Primary periodontic lesion with secondary endodontic involvement

- True combined lesion

The most important factor in the treatment is a correct diagnosis which is achieved by careful history taking, examination and the use of special tests. Specific things to look for in the history include past disease, trauma and pain. The teeth are examined for abnormalities such as caries, defective restorations, erosions, abrasions, cracks, fractures, and discolorations.

Advanced lesions and true-combined lesions are difficult to differentiate between and therefore, where doubt exists they should be considered as an endodontic lesion in origin.

\section{CASE REPORT}

A 35 year old female patient reported to Department of Periodontics and Oral Implantology, UCDS, Bhairahawa, Nepal complaining of mobility, sensitivity and spacing of upper front teeth. The medical history was noncontributory. Intra oral examination revealed midline diastema with attrition of lower anteriors. Probing depth of $8 \mathrm{~mm}$ was observed mesial to tooth 11. Palpation revealed tenderness on percussion and grade II mobility on 11 .

Radiographic examination revealed radiolucency at the apex with widening of PDL space and severe angular bone defect ( three walled defect)with normal crown. Radiographic examination of 12 shows periapical pathology and widening of PDL space with normal crown. Initially root canal therapy followed by periodontal regenerative surgery was suggested. The patient was then referred to Department of Conservative dentistry to check for tooth vitality. Electric pulp testing was done which confirmed that the both 11 and 12 were non-vital. The clinical and radiographic findings suggested of a combined endoperio lesion of 11 and 12 due to trauma from occlusion. Initially root canal therapy followed by periodontal surgery was suggested.

Figure 2: (A) preoperative photograph, (B) Periodontal probing depth was $8 \mathrm{~mm}$ mesially

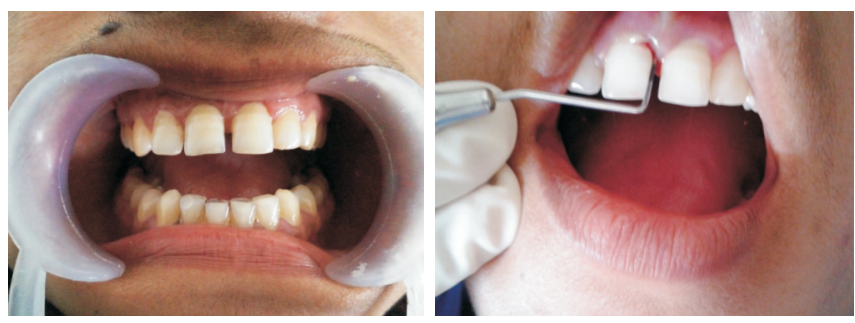

\section{Endodontic management}

Endodontic access cavity was prepared on tooth 11 and working length was determined. Cleaning and shaping of the root canal was performed by using conventional stainless steel $\mathrm{K}$ files with a step back technique under copious irrigation with saline, $3 \%$ sodium hypochlorite solution and 17\% EDTA. Apical preparation was done upto no. $30 \mathrm{~K}$ file and coronal preparation upto no. $55 \mathrm{~K}$ file. The canal was dried and an inter appointment dressing with calcium hydroxide was placed and a temporary filling was placed in the access cavity. Occlusal reduction of lower anteriors was done to relieve the trauma from occlusion. The patient was recalled after 10 days and the canal was obturated and permanently restored with glass ionomer cement. Following this, the patient was recalled after1 month for periodontal therapy.

Figure 3: (A) Preoperative, (B) Mastercone Xray, (C) Post obturation Xray

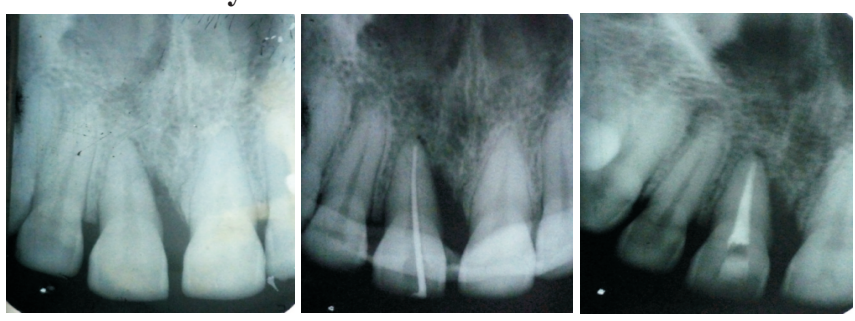

\section{Surgical procedure}

When the patient reported after one month, the mobility was reduced and the tooth was asymptomatic. However, the probing depth was persistant and hence periodontal therapy was initiated.

The area selected for surgery was anesthetized using lignocaine with adrenaline 1:150,000. A full thickness flap 
was raised at the buccal aspect following intracrevicular incision extending from distal of 12 to distal of 21 . And a vertical releasing incision extending into the alveolar mucosa not closer than one tooth to the involved area, i.e. 11. Full thickness flap was raised. After reflection thorough degranulation and debridement was done at the defect area using Gracey's curette \# 1-2 and 3-4. Also thorough scaling and root planning was carried out. The flap was then positioned coronally, there by resulting in complete coverage of the defect.

Primary soft tissue closure of the flap was done with non absorbable black silk (30) suture using interrupted suturing technique and periodontal dressing was placed.

Figure 4: (A) Full thickness flap raised, (B) Periodontal dressing given after surgery
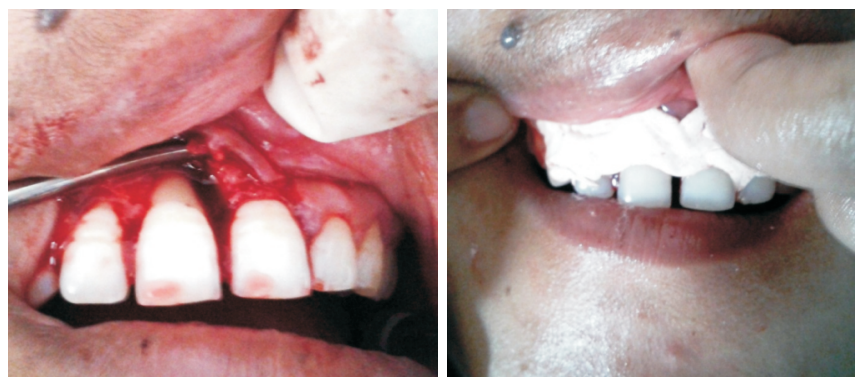

The sutures were removed 7 days after surgery . The patient was put on regular recall at 1 and 3 months. Following the three months follow up the tooth was asymptomatic with reduction in mobility to grade $\mathrm{I}$. The probing depth was $3 \mathrm{~mm}$ all around the tooth and no inflammation was seen on soft tissues. Radiographic examination revealed evidence of regeneration of the periradicular bone.

\section{Figure 5: (A) 1 month follow up, (B) 3 month follow up}
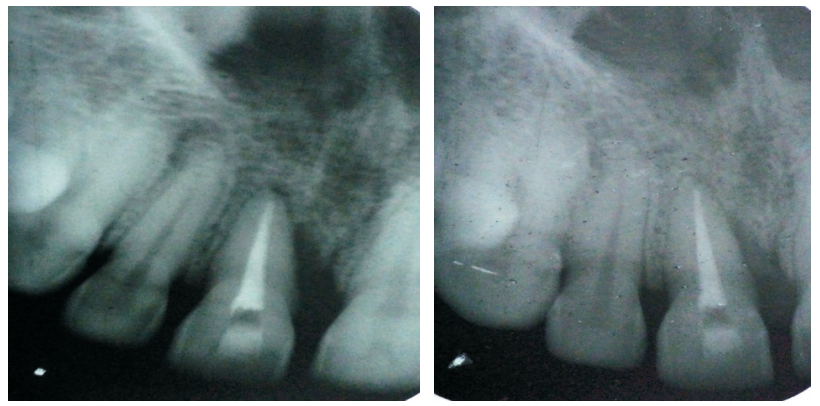

Figure 6: The probing depth was found to reduce by $3 \mathrm{~mm}$.

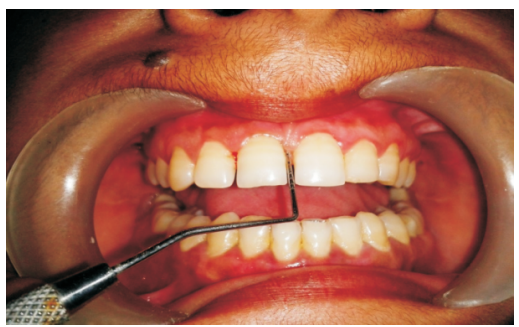

\section{Aesthetic correction}

At the third month follow up, substantial amount of bone fill mesial to 11 was observed which was sufficient to stabilize the tooth and reduce its mobility. Aesthetic correction of midline diastemsa was, hence, initiated. Under rubber dam isolation, tooth preparation was done, the enamel surface of both teeth were etched for 30 seconds, after which the adhesive bonding agent was placed and cured. Lingual wall was build up using flowable composite (Tetric Ivoflow, Ivoclar Vivadent) A3 shade and labially A2 shade (Beautifil, Shofu).

\section{Figure 7: (A)Acid etching, (B)After finishing and polishing}
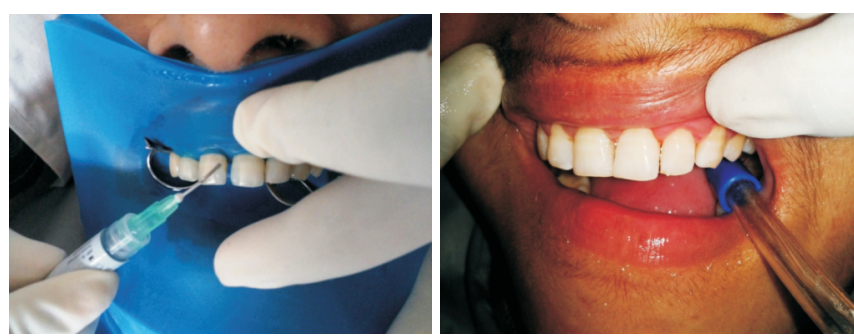

\section{DISCUSSION}

When a definitive diagnosis in the case of an endoperio lesion cannot be attained, it may be prudent to initiate either of treatment modalities and hope for repair. However, this could be overcome by proper history taking and sequential treatment planning. Analyzing a series of retrospective studies, Blomlof et al concluded that endodontic infection promotes periodontal pocket formation and should be regarded as a risk factor in periodontitis progression.

In this report the pulp vitality test which showed the non vital nature of the tooth was a pivoting finding suggesting the primary endodontic involvement. Generally, in a case of combined endoperio lesion, an adequate endodontic therapy would result in healing of the endodontic component, and the prognosis would finally depend on the efficacy of periodontal repair or regeneration initiated by either of treatment procedures. ${ }^{2}$ In this case, calcium hydroxide was used as the intracranial inter-appointment dressing to disinfect the root canal system further and to evaluate the improvement of the surrounding tissues at the second appointment, at which time it was decided to fill both teeth.

In this case following endodontic treatment periodontal lesion did not subside after 1 month evaluation. This confirmed a secondary periodontal involvement which needed periodontal intervention. Johnson et al ${ }^{8}$ presented Periodontal Treatment Needs System (PTNS) which is based on the motivation and instruction in effective oral hygiene measures, subgingival debridement of the root surfaces of periodontally involved teeth and debridement facilitated by surgery. 
Obtaining periodontal regeneration has always been a major challenge and several approaches to periodontal regeneration have been used throughout the years. If the objective is to increase the clinical attachment level, non surgical therapy is of greater benefit for shallow (1-3mm) and moderate (4-6mm) pockets ${ }^{3}$, and open flap debridement for deep pockets $(>6 \mathrm{~mm})$. ${ }^{9}$ The success rate of the endodontic-periodontal combined lesion without a concomitant regenerative procedure has been reported to range from $27 \%-37 \%{ }^{4}$

Wang and Boyapati ${ }^{7}$ suggested 4 factors, the so-called PASS principle, that are critical for predictable bone regeneration: primary wound closure, angiogenesis as a blood supply and source of undifferentiated mesenchymal cells, space maintenance, and stability of the wound. Some of the patient factors that might contribute to positive outcome includes the good plaque control, compliance, nonsmoking, anti-infective therapy and systemic health. Other factors that might also negatively affect the healing process include occlusal trauma, improper surgical technique (such as excessive flap tension), early mechanical disruption, and contamination during surgery. Occlusal trauma in this case was reduced by therapeutic grinding of lower anteriors.

The choice of the therapy also depends on evaluation of adverse effects and patient centered outcomes such as patient discomfort, apprehension, aesthetic consideration and the cost effectiveness of the therapy. In this case the patient was unwilling for endodontic management of assymptomatic, non vital tooth 12 as well as regenerative procedures such as bone graft, considering the cost. However, from clinical and radiographic findings, the result of this combined technique was quite impressive, resulting in a significant reduction of probing depth and substantial bone fill.

\section{CONCLUSION}

The healing of an endodontic lesion is highly predictable but the repair or regeneration of periodontal tissue is questionable if associated with it. Endodontic therapy mostly should precede periodontal pocket elimination procedures in the case of a primary endo and secondary perio involvement, however, endodontic therapy would result only in resolution of the endodontic component of involvement and would have a little effect on the periodontal lesion. This case report demonstrates that proper diagnosis, followed by removal of etiological factors and utilizing the combined treatment modalities will restore health and function to the teeth with severe attachment loss caused by an endo-perio lesion.

\section{REFERENCES}

1. Bender IB, Seltzer S. The effect of periodontal disease on the pulp.Oral surg Oral med Oral path 1972: 33: 458-474.

2. Narang S, Narang A, Gupta R. A sequential approach in treatment of perio endo lesion. J.Indian Society of periodontology 2011, Vol 15, 177-180.

3. Lovadal A, Arno O, Schei O, Waerhaug J. Combined effect of subgingival scaling and controlled oral hygiene on the incidence of gingivitis. Acta Odontol Scand, 1961;19:537555 .

4. Hirsch JM, Ahlström U, Henrikson PÄ, Heyden G, Peterson LE. Periapical surgery. International Journal of Oral Surgery. 1979;8(3): 173-85.

5. Jansson L, Ehnevid H, Lindskog S, Blomlöf L. Relationship between periapical and periodontal status. Journal of Clinical Periodontology. 1993;20(2):117-23.

6. Shenoy N, Shenoy A. Endo-perio lesion: diagnosis and clinical consideration. Indian Journal of Dental Research, 2010;21(4): 579-585.

7. Wang HL, Boyapati L. "PASS" principles for predictable bone regeneration. Implant Dent 2006;15:8-17.

8. Johnson JR, Gjermo P, Bellini HD. A System to classify the need for periodontal treatment. Acta Odontol Scand, 1973;31:297-305.

9. Kinena DF, Lindhe J, Trombelli L. Clinical periodontology and implant dentistry. $5^{\text {th }}$ edn. Oxford Blackwell publishing Ltd. 2008, 420-427.

10. Gjermo PE, Grytten J. Cost effectiveness of various treatment modalities for adult chronic periodontitis. Periodontology 2000, vol:51; 2009:269-275

11. Verma PK, Srivastava R, Gupta KK, Srivastava A. Combined endodontic-Periodontal lesion: A clinical dilemma. Journal of interdisciplinary dentistry, $2011 ; 1: 2: 119-124$.

12. Kumar R, Patil S, Hoshing U, Medha A, Mahaparale R. Nonsurgical endodontic management of the combined Endoperio lesion. International Journal Of Dental Clinics 2011:3(2):82-84

13. Lisa JA, Heitz M. How effective surgical therapy compared with non surgical debridement. Periodontology 2000; 37 :2005: 72-87. 